\title{
Valutazione Del Benessere Psico-Fisico Nell'aderenza Terapeutica Nelle Donne Con Malattia Renale Policistica Autosomica Dominante: Uno Studio Osservazionale
}

Giornale di Tecniche Nefrologiche e Dialitiche 2019, Vol. 31(3) 160-166

(c) The Author(s) 2019

Article reuse guidelines:

sagepub.com/journals-permissions DOI: 10.1 | 177/03949362 I9858903 journals.sagepub.com/home/gtn

@SAGE

\author{
Elena Brioni 1,2, Cristiano Magnaghi3,2, Giulia Bruna Delli Zotti,2, \\ Eleonora Sangiovanni ${ }^{5}$, Maria Teresa Sciarrone Alibrandi 6 , \\ Luigi Apuzzo 7,2 , Paolo Manunta ${ }^{8}$, Francesco Logias 9 \\ e Francesco Burrai ${ }^{10}$
}

\begin{abstract}
Evaluation Of The Psychophysical Well-Being In The Compliance Of Women With Autosomal Dominant Policystic Kidney Disease: An Observational Study

BACKGROUND: Autosomal dominant polycystic kidney disease is the most common inherited renal disease and affects less than I every 400-I,000 people. There are many effective treatments, including blood pressure management, physical activity, low sodium diet and hydration. Therapeutic education is part of a patient's care and treatment. This approach is an essential strategy in order to face the current healthcare scenario, in which the number of people affected by chronic diseases is progressively increasing. OBJECTIVES: This article aims to analyze the effect of therapeutic education in patients with ADPKD, the level of adherence to pharmacological therapy and their compliance to dietetic and lifestyle recommendations as part of a nursing-led education. METHODS: This is a prospective, longitudinal, observational pilot study. The following measurements were used: Kidney Disease Quality of life - Short Form, Hospital Anxiety and Depression Scale, Body Uneasiness Test. At the T0 visit, a nurse selected patients and carried out a personalized educational intervention with the aims of adhering to drug therapies, monitoring blood pressure and dietary behavior (physical activity and water intake). At the TI visit, patients performed psychological tests. At the T2 visit, the following evaluations were performed: a psychological interview together with the delivery and evaluation of the tests performed, an interview with the nurse to evaluate the adherence to the prescriptions, and a control of parameters such as physical activity, diet, water intake, drug therapy, and blood pressure. RESULTS: Therapeutic education can have a positive
\end{abstract}

'RMN, RN. Infermiera di ricerca senior. Genomica delle malattie renali ed ipertensione, Ospedale San Raffaele di Milano, Milano, Italy

${ }^{2}$ Ricercatore centro di ricerca SIAN, Bologna, Italy

${ }^{3}$ RMN, RN. Infermiere di Dialisi. Nefrologia e Dialisi. Ospedale San

Raffaele Milano, Milano, Italy

${ }^{4}$ Psicologo e psicoterapeuta Servizio sanitario di psicologia, IRCCS

Ospedale San Raffaele, Milano, Italy

${ }^{5}$ Psicologo Servizio sanitario di psicologia, IRCCS Ospedale San

Raffaele, Milano, Italy

${ }^{6} M D$ Specialista in Nefrologia, Dialisi ed Ipertensione, IRCCS Ospedale

San Raffaele, Milano, Italy

${ }^{7}$ MSC, RN. Ricercatore centro di ricerca SIAN, Bologna, Italy
${ }^{8} \mathrm{PhD}$, Prof.Cattedra di Nefrologia Università San Raffaele. Genomica delle malattie renali e laboratorio di ipertensione. Ospedale IRCCS San Raffaele, Milano, Italy

9MD, Direttore Nefrologia e Dialisi, Ospedale San Francesco, Nuoro, ATS Sardegna, Italy

${ }^{10} \mathrm{PhD}$, Formazione, Ricerca e Cambiamento Organizzativo. ATS Sardegna, Italy

\section{Corrispondenza:}

Francesco Burrai, PhD, SC Formazione, ricerca e cambiamenti organizzativo. ATS Sardegna, Via Strada Statale 200, 07100, Sassari, Italy. E-mail: francesco.burrai@atssardegna.it 
impact on patients' health by improving adherence to the pharmacological therapy, diet and lifestyle. CONCLUSIONS: Therapeutic education improve the patient's knowledge, treatments and correct behaviors as well as promotes an independent management of the disease. Through an educational intervention, the patient acquires the ability and the awareness to modify the wrong behaviors and to guarantee a balance between his needs and the pathology, thus improving the quality of life.

\section{Keywords}

Therapeutic adherence, Autosomal dominant polycystic kidney disease, Lifestyle, Therapeutic education, Physical activity, Diet

\section{Introduzione}

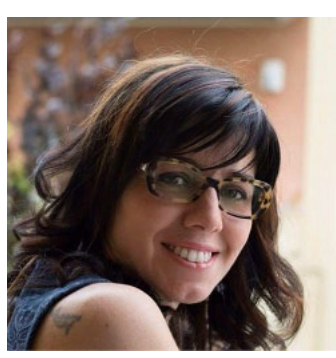

La malattia renale policistica autosomica dominante (Autosomal dominant polycystic kidney disease, $\mathrm{ADPKD}$ ) è la più comune malattia renale ereditaria, che si verifica in circa 1 ogni 400-1,000 persone. " "Autosomica dominante" significa che ogni figlio di un genitore affetto ha una probabilità del $50 \%$ di ereditare la malattia. La malattia è caratterizzata da un decorso subclinico prolungato di progressiva espansione delle cisti renali, con conseguente e massiccio aumento del volume del rene, ridotta funzionalità e progressione della malattia fino allo stadio finale. ${ }^{2}$

La malattia si può manifestare anche con la crescita di cisti epatiche che aumentano tendenzialmente con l'età e sono più frequenti e più grandi nelle donne che nell'uomo. Queste cisti non causano una disfunzione epatica ma un notevole aumento delle dimensioni del fegato, che può causare una compressione degli organi $\operatorname{circostanti}^{3} \mathrm{e}$ talvolta dell'organo stesso, arrivando raramente a dover ricorrere ad un trapianto di fegato.

La sintomatologia ansioso-depressiva è molto frequente nei pazienti nefropatici di sesso femminile e sembra essere correlata a una ridotta aspettativa di vita. ${ }^{4} \mathrm{La}$ malattia renale allo stadio terminale (end-stage renal disease, ESRD) è una condizione somatica grave e irreversibile, ed il 'calvario' della malattia può minare $\mathrm{i}$ marcatori interni di identità dei pazienti, preda di angosce insopportabili, esponendoli così ad impazienza e ad un'identità vacillante. ${ }^{5}$

Le persone con una malattia cronica subiscono continui adattamenti psicologici e comportamentali in relazione ai vari cambiamenti imposti dalla loro patologia. L'impatto che può avere la diagnosi di malattia cronica sull'individuo ha molteplici effetti simultaneamente e a diversi livelli: personale, interpersonale, familiare, sociale e occupazionale. ${ }^{6}$

L'ADPKD ha conseguenze sulla percezione dell'immagine corporea, infatti i pazienti che ne sono colpiti spesso segnalano un forte disagio a causa di gonfiore addominale cronico, sintomo presente in pazienti di entrambi i sessi, ma particolarmente fastidioso nelle donne. ${ }^{7}$ Si nota anche che le deformità fisiche dovute a malattie renali possono portare ad insicurezza e a ripercussioni sull'autostima. ${ }^{8}$

Disturbi d'ansia sono costantemente associati alla percezione della qualità della vita dei pazienti affetti da ESRD, la percezione della qualità della vita è stata associata a esiti clinici comprendenti l'aderenza comportamentale del paziente, lo stato nutrizionale e la mortalità. È inoltre associata ad una diminuzione dello stato di salute e ad una qualità della vita sostanzialmente inferiore alla salute; questo persiste nel tempo ed è anche associato ad un maggiore uso di servizi di assistenza sanitari. ${ }^{9}$ I trattamenti sono spesso complessi e richiedono una gestione attiva e personalizzata, rendendo arduo il raggiungimento e il mantenimento di un'autocura ottimale. Infatti, oltre al requisito per i pazienti di assumere farmaci come prescritto, viene a loro anche richiesto di adottare una serie di modelli comportamentali no solo per gestire e ottenere un buon controllo e monitoraggio dei sintomi, evitando così fattori aggravanti (quali l'ipertensione $\mathrm{e}$ l'infezione del tratto urinario), ma anche per modificare lo stile di vita. Alcuni trattamenti che possono attenuare la progressione della malattia renale sono: controllo della pressione arteriosa, esercizio fisico, dieta a basso contenuto di sale, assunzione di molta quantità di acqua. ${ }^{10}$

L'aderenza implica la scelta attiva del paziente di seguire le raccomandazioni mediche piuttosto che la cooperazione passiva di obbedienza; inoltre, l'aderenza è associata a diversi fattori, che possono essere correlati con i farmaci prescritti come ad esempio: il numero di farmaci da assumere, le possibili difficoltà nella manipolazione del medicamento, i regimi complessi, gli effetti collaterali e il costo. $^{11}$ Esistono approcci terapeutici efficaci che potrebbero in alcuni casi prevenire l'ESRD e, in molti altri, rallentare la sua progressione.

L'educazione terapeutica del paziente è un'istruzione gestita da professionisti sanitari addestrati all'educazione dei pazienti e progettata per consentire a un paziente (o a un gruppo di pazienti e famiglie) di gestire il trattamento della loro condizione e di prevenire complicazioni evitabili, mantenendo o migliorando la qualità della vita. Il suo scopo principale è quello di produrre un effetto terapeutico aggiuntivo a quello di tutti gli altri interventi (farmacologici, terapie fisiche, ecc.). ${ }^{12}$ 
Inoltre, un programma di educazione terapeutica può ottenere una migliore qualità della vita in una condizione cronica, perché le esigenze biomediche, educazionali e psicosociali del paziente vengono identificate portandolo alla piena consapevolezza della sua malattia: ${ }^{13}$ viene così valorizzata la sua auto-cura. ${ }^{14}$

La comprensione della complessità della malattia e del trattamento, tuttavia, insieme con la comprensione dei benefici a medio-lungo termine relativi al trattamento specifico rappresentano il miglior suggerimento per il paziente e quindi migliorano l'aderenza. ${ }^{15}$

\section{Obiettivo}

L'obiettivo principale dello studio è valutare gli effetti dell'educazione terapeutica nei pazienti con ADPKD e CKD. L'istruzione può avere un effetto positivo sul livello di aderenza alla terapia farmacologica (effetti collaterali, rischi, benefici, modalità di somministrazione), sulla dieta, sul controllo della pressione arteriosa e su stili di vita sani, con un possibile miglioramento dell'empowerment del paziente. L'obiettivo secondario è quello di valutare in che modo un approccio educativo può offrire più conoscenza e consapevolezza e ridurre i sintomi dell'ansia acquisendo maggiore autostima e rafforzando la percezione dell'immagine del proprio corpo. Questi obiettivi sono stati scelti perché le variabili dipendenti sono fortemente influenzate dall'educazione terapeutica con l'obiettivo di rendere il paziente attivo nel processo assistenziale attraverso un approccio multidisciplinare per migliorare la qualità della vita.

\section{Metodi}

Questo è uno studio pilota prospettico longitudinale osservazionale.

È stato considerato un campione convenzionale di 40 pazienti, donne affette da ADPKD afferenti all' ambulatorio specialistico dell'ADPKD dell'Ospedale San Raffaele di Milano durante il periodo dal 01/09/2017 al 01/06/2018. La metodologia di campionamento era consecutiva, non probabilistica e non randomizzata. I criteri di inclusione comprendevano diagnosi confermata di ADPKD, età da 18 a 65 anni, tutte le fasi di CKD e capacità di fornire il consenso informato. La scelta di considerare solo un campione femminile è stata stabilita in quanto la formazione delle cisti epatiche nelle donne ha un decorso più aggressivo a causa delle gravidanze e dell'esposizione agli estrogeni. Pertanto, le donne sono più soggette a complicazioni come infezioni, ascite e ostruzione venosa epatica. Nella rappresentazione epatica della malattia policistica è presente un'alterazione fisiognomica significativa (aumento della circonferenza addominale), ridotta mobilità, diminuzione della qualità della vita $\mathrm{e}$ dell'immagine corporea. ${ }^{16}$
Sono stati esclusi pazienti di sesso maschile, pazienti con disturbi cognitivi, pazienti in dialisi o pazienti già sottoposti a trapianto renale.

\section{Misurazioni}

\section{Informazioni demografiche e storia medica}

Per le informazioni demografiche e la storia medica è stata utilizzata una scheda raccolta dati (CRF). I dati raccolti includono variabili sociodemografiche e cliniche quali l'età, lo stato civile, il livello di istruzione, l'occupazione, il numero di figli, le comorbidità dell'indice di massa corporea (BMI), l'assunzione di terapie farmacologiche (incluso il Tolvaptan) e i valori di pressione arteriosa. La pressione arteriosa è stata misurata in $\mathrm{mm} / \mathrm{hg}$ con Omron M6 Comfort IT Automatic Digital Blood Pressure Monitor (OMRON Healthcare Europe B.V., Hoofddrp, The Netherlands). Sono stati inoltre raccolti i dati rispetto all'attività fisica, alle norme dietetiche rispetto ad una dieta a basso contenuto di sale e a basso contenuto proteico, all'assunzione di acqua, al controllo della pressione arteriosa, alla terapia prescritta e alla puntualità rispetto alle scadenze previste per le visite mediche. Queste indagini sono state registrate da parte dell'infermiere ad ogni visita e qualora la paziente non si fosse presentata, è stata sollecitato telefonicamente. Riguardo allo stato di gravidanza, nelle donne in menopausa queste domande sono state riferite al loro periodo fecondo e a quando si sono trovate nella situazione di pianificare una gravidanza.

\section{Qualità della vita}

The KidneyDisease Quality ofLife-ShortForm (KDQOL-SF) è stato utilizzato per misurare la qualità della vita. Il KDQOL-SF è un questionario convalidato in italiano ${ }^{17}$ per la valutazione della qualità della vita auto-percepita.

\section{Ansia}

The Hospital Anxiety and Depression Scale (HADS) è stato utilizzato per misurare l'ansia; ${ }^{18}$ è un questionario autosomministrato specifico per la valutazione della sintomatologia ansiosa e depressiva del paziente.

\section{Immagine del corpo}

The Body Uneasiness Test (BUT) è stato utilizzato per misurare l'immagine corporea ${ }^{19}$ e per valutare un'immagine corporea disturbata.

\section{Aderenza}

L'aderenza è stata definita controllando le variabili: partecipazione alla visita medica, attività fisica, assunzione 
di acqua, terapia farmacologica, controllo della pressione arteriosa e aderenza ai modelli dietetici. Infine, la totale compliance alla cura è stata valutata per tutte le pazienti che avevano mostrato aderenza a tutte le variabili rispetto a quelle che non erano state aderenti anche a una sola variabile.

\section{Raccolta dati}

Questo studio ha avuto 3 visite (T0, T1, T2) e ciascuna visita è durata un'ora.

Durante le visita T0, un'infermiera sottoponeva a screening le pazienti di sesso femminile con ADPKD che sono state indirizzate all'ambulatorio specialistico di malattia policistica dell'ospedale San Raffaele di Milano. L'infermiera contattava quindi le potenziali candidate per spiegare lo studio e offrire loro l'opportunità di firmare il modulo di consenso informato. Durante questa visita l'infermiera, attraverso un'intervista, raccoglieva tutte le informazioni demografiche e i dati anamnestici, compilava la scheda raccolta dati e controllava la pressione arteriosa. In questa visita, l'infermiera si occupava anche di classificare lo stadio di funzionalità renale del paziente secondo eGFR, ${ }^{3}$ distinti in stadi da G1 a G5. Inoltre, l'infermiera eseguiva un intervento educativo standardizzato per istruire la paziente sull'assunzione delle terapie farmacologiche (in particolare il rispetto dell'orario e della dose) utilizzando anche consigli scritti e altri ausili per la comunicazione. La paziente veniva informata sulla dieta corretta da seguire (dieta a basso contenuto di sodio e/o ipoproteica secondo prescrizione medica) e sull'importanza di pianificare un programma di attività fisica costante. La paziente veniva sollecitata ad eseguire il monitoraggio domiciliare della pressione e ad assumere un'adeguata quantità di liquidi. L'intervento terapeutico era supportato da materiale informativo consegnato alla paziente al termine della visita e l'infermiera esortava la paziente a porre qualsiasi domanda che ritenesse necessaria chiedendole anche di ripetere le informazioni essenziali fornite. Durante la visita T1, il paziente compilava tutti i questionari psicologici (KDQOL-SF, HADS e BUT).

Alla terza visita (T2), dopo un mese dalla visita T0, l'infermiera e lo psicologo fornivano un feedback sulla valutazione dei test eseguiti; inoltre, l'infermiera si occupava di intervistare le pazienti per valutare l'aderenza alle prescrizioni farmacologiche, all'attività fisica, alla dieta, al controllo della pressione arteriosa e all'adeguato introito idrico, spiegando loro anche l'importanza di seguire puntuali controlli clinici (esami e visite) con l'obiettivo di monitorare un eventuale peggioramento della patologia e intervenire tempestivamente.

Queste informazioni rilevate venivano registrate nella scheda raccolta dati delle paziente.

\section{Considerazioni Etiche}

Questo studio rispetta la Dichiarazione di Helsinki ed è stato approvato dal comitato di revisione istituzionale locale e dal comitato etico dell'Ospedale San Raffaele.

\section{Analisi Statistica}

I fattori categoriali sono stati riassunti utilizzando frequenze e percentuali, mentre le misure continue sono state descritte utilizzando medie e deviazioni standard per le variabili normalmente distribuite, e mediane e quartili per le misure non normalmente distribuite. Per il confronto delle caratteristiche del paziente, sono stati utilizzati i t-test di Student come appropriato. Le differenze psicologiche di conformità ("conforme" - G1 e "non conforme" - G2) sono state analizzate utilizzando il test t-test indipendente.

Le correlazioni di Pearson sono state utilizzate per la correlazione tra caratteristiche psicologiche e livelli di eGFR. Tutte le statistiche erano a due lati e il livello di significatività impostato $\mathrm{a} p<0.05$. Le analisi sono state eseguite utilizzando SPSS тм (IMB Statistics per Windows versione 20 ).

\section{Risultati}

Un campione totale di 40 pazienti, donne con ADPKD, è stato reclutato per lo studio, tuttavia questo studio è stato condotto su un campione di 37 pazienti in quanto vi è stato un abbandono di 3 pazienti. Questi 3 pazienti hanno chiesto di lasciare lo studio e hanno ritirato il loro consenso informato.

L'età media di tutte le pazienti è $44.39 \pm 9.5$. Lo stato civile delle donne in studio ha mostrato che il $62.2 \%$ era sposato, il $13.5 \%$ era divorziato e il $24.3 \%$ era single. Il $73.0 \%$ delle donne iscritte aveva avuto una gravidanza. Riguardo alle condizioni mediche concomitanti, il 67.6\% aveva l'ipertensione, il $10.8 \%$ aveva una storia di aneurismi cerebrali, il $10.8 \%$ presentava difetti valvolari cardiaci e il $51.4 \%$ aveva infezioni del tratto urinario. Il $64.9 \%$ aveva una familiarità per $\mathrm{ADPKD}$, il $32.4 \%$ aveva parenti in dialisi e il $27.5 \%$ aveva parenti sottoposti a trapianto renale; inoltre il $21.6 \%$ aveva preso terapia con Tolvaptan. Riguardo la componente psicologica remota, il $29.7 \%$ ha seguito interviste psicologiche prima dell'arruolamento, ma solo il $2.7 \%$ ha assunto un farmaco psichiatrico. Per quanto riguarda l'aderenza, i risultati hanno mostrato un'adesione all'attività fisica del $45.9 \%$, agli schemi dietetici del $75.5 \%$, all'assunzione di acqua dell' $86.5 \%$, ai controlli pressori del $75.7 \%$, agli esami medici del $70.3 \%$ e al rispetto delle indicazioni fornite per la terapia farmacologica $100 \%$. Le caratteristiche demografiche e cliniche delle pazienti sono mostrate nella Tabella 1.

Al fine di determinare lo stadio di insufficienza renale delle pazienti, il valore utilizzato è stato quello del tasso di 
Tabella I. Informazionini demografiche e storia medica.

\begin{tabular}{|c|c|c|}
\hline \multirow[t]{2}{*}{ Variabile indipendente } & \multicolumn{2}{|l|}{ Campione } \\
\hline & \multicolumn{2}{|l|}{$\mathbf{N}=\mathbf{3 7}$} \\
\hline Età (media $\pm D S)$ & \multicolumn{2}{|l|}{$44.39 \pm 9.5$} \\
\hline \multicolumn{3}{|l|}{ Stato civile, n (\%) } \\
\hline Celibe & 9 & $(24.3 \%)$ \\
\hline Coniugato & 23 & $(62.2 \%)$ \\
\hline Divorziato & 5 & (13.5\%) \\
\hline \multicolumn{3}{|l|}{ Istruzione scolastica, n (\%) } \\
\hline Analfabeta & 0 & 0 \\
\hline Scuola elementare di primo grado & 2 & $(5.4 \%)$ \\
\hline Scuola secondaria di primo grado & 3 & $(8.1 \%)$ \\
\hline Scuola secondaria di secondo grado & 18 & $(48.6 \%)$ \\
\hline Università & 14 & $(37.8)$ \\
\hline \multicolumn{3}{|l|}{ Condizione di lavoro, n (\%) } \\
\hline Lavoro (o impiegato) & 31 & $(83.8 \%)$ \\
\hline Non occupato (o disoccupato) & 6 & (16.2\%) \\
\hline Q_I sì & 16 & (43.2\%) \\
\hline Q_I NO & 21 & $(56.8 \%)$ \\
\hline Q_2 sì & 20 & (54.1\%) \\
\hline Q_2 NO & 17 & $(45.9 \%)$ \\
\hline \multicolumn{3}{|l|}{ Condizione familiare, n (\%) } \\
\hline Bambino Sì & 27 & $(73.0 \%)$ \\
\hline Bambino no & 10 & 27 \\
\hline I bambino/a & 12 & $(32.4 \%)$ \\
\hline 2 bambini & II & (29.7\%) \\
\hline 3 bambini e ragazzi & 2 & $(5.4 \%)$ \\
\hline 4 bambini & I & $(2.7 \%)$ \\
\hline 5 bambini e ragazzi & $\mathrm{I}$ & $(2.7 \%)$ \\
\hline Risorse famigliari & 22 & $(59.5 \%)$ \\
\hline Risorse sociali & 8 & $(21.6 \%)$ \\
\hline Risorse culturali & 8 & $(21.6 \%)$ \\
\hline Risorse spirituali & 7 & (18.9\%) \\
\hline \multicolumn{3}{|l|}{ Condizione medica, n (\%) } \\
\hline Visite psicologiche & II & $(29.7 \%)$ \\
\hline $\begin{array}{l}\text { Partecipazione al protocollo } \\
\text { Tolvaptan }\end{array}$ & 8 & $(21.6 \%)$ \\
\hline Farmaci psichiatrici & $\mathrm{I}$ & $(2.7 \%)$ \\
\hline Malattie concomitanti & $32 \mathrm{a}$ & $(86.5 \%)$ \\
\hline Ipertensione & 25 & $(67.6 \%)$ \\
\hline Aneurismi & 4 & $(10.8 \%)$ \\
\hline Difetti valvolari & 4 & $(10.8 \%)$ \\
\hline Infezioni del tratto urinario & 19 & (5I.4\%) \\
\hline Familiarità con ADPKD & 24 & (64.9\%) \\
\hline Analisi genetica & 18 & (48.6\%) \\
\hline Parenti in dialisi & 12 & (32.4\%) \\
\hline Parenti con trapianto renale & 10 & $(27.5 \%)$ \\
\hline Conformità totale & 12 & (32.4\%) \\
\hline Attività fisica & 17 & (45.9\%) \\
\hline Esami medici & 26 & (70.3\%) \\
\hline Modelli dietetici di aderenza & 28 & (75.5\%) \\
\hline Assunzione d'acqua & $32 \mathrm{a}$ & (86.5\%) \\
\hline Terapia farmacologica & $37 \mathrm{a}$ & $(100 \%)$ \\
\hline Controlli pressione arteriosa & 28 & (75.7\%) \\
\hline \multicolumn{3}{|l|}{ BMI, n (\%) } \\
\hline Peso normale $>$ I8.5 $(<25.0)$ & 23 & $(62.2 \%)$ \\
\hline In sovrappeso $>25.0$ & 14 & (37.8\%) \\
\hline
\end{tabular}

Tabella 2. Classificazione eGFR.

\begin{tabular}{llc}
\hline Stadio & $\begin{array}{l}\text { Campione } \\
\mathrm{n}=37 \\
\mathrm{n}(\%)\end{array}$ & $\begin{array}{l}\text { eGFR } \\
\left(\mathrm{mL} / \mathrm{min} / 1.73 \mathrm{~m}^{2}\right) \\
\text { Range }\end{array}$ \\
\hline 1 & $11(29.7 \%)$ & $\geqslant 90$ \\
2 & $8(21.6 \%)$ & $60-89$ \\
3 & $10(27.0 \%)$ & $30-59$ \\
4 & $6(16.2 \%)$ & $15-29$ \\
5 & $2(5.4 \%)$ & $<15$ \\
\hline
\end{tabular}

Tabella 3. HADS, KDQOL-SF e BUT.

\begin{tabular}{ll}
\hline Questionario & Campione \\
\cline { 2 - 2 } & $\begin{array}{l}\mathrm{n}=37 \\
\text { (media } \pm \mathrm{DS} \text { ) }\end{array}$ \\
\hline HADS & \\
HADS - ansia & $6.00 \pm 3.75$ \\
HADS - depressione & $3.86 \pm 3.50$ \\
KDQOL-SF & \\
Sintomi & $78.01 \pm 14.45$ \\
Effetti della malattia & $75.51 \pm 18.75$ \\
Peso della malattia & $68.42 \pm 23.25$ \\
Lavoro & $90.54 \pm 19.85$ \\
Funzioni cognitive & $81.08 \pm 19.34$ \\
Interazioni sociali & $63.76 \pm 8.71$ \\
Funzioni sessuali & $90.54 \pm 24.01$ \\
Sonno & $67.54 \pm 11.71$ \\
Assistenza sociale & $74.29 \pm 26.52$ \\
Funzionamento fisico & $77.64 \pm 19.36$ \\
Funzionamento del ruolo fisico & $65.27 \pm 41.78$ \\
Dolore & $75.70 \pm 29.26$ \\
Stato di salute generale & $56.76 \pm 13.02$ \\
Benessere emotivo & $69.84 \pm 17.71$ \\
Funzionamento del ruolo emotivo & $73.86 \pm 35.27$ \\
Funzionamento sociale & $75.35 \pm 24.54$ \\
Energia & $55.81 \pm 18.09$ \\
BUT & \\
GSI (indice di gravità globale) & $0.95 \pm 0.64$ \\
WP (fobia del peso) & $1.23 \pm 0.78$ \\
BIC (problema dell'immagine corporea) & $1.36 \pm 0.92$ \\
A (elusione) & $0.47 \pm 0.67$ \\
CSM (autocontrollo compulsivo) & $0.76 \pm 0.64$ \\
D (depersonalizzazione) & $0.48 \pm 0.63$ \\
PSDI (indice di distress psicologico dei & $2.09 \pm 0.70$ \\
sintomi) & \\
\hline & \\
\hline
\end{tabular}

filtrazione glomerulare stimato (estimated glomerular filtration rate, eGFR). La classificazione eGFR è mostrata nella Tabella 2.

È stata eseguita un'analisi descrittiva dei punteggi medi dei questionari psicologici.

I punteggi dei questionari HADS, KDQOL-SF e BUT sono mostrati nella Tabella 3. 
Tabella 4. Caratteristiche psicologiche secondo l'aderenza.

\begin{tabular}{|c|c|c|c|c|}
\hline \multirow[t]{2}{*}{ Variabile } & \multirow{2}{*}{$\frac{\text { Aderenza }}{\mathrm{GI}}$} & \multirow{2}{*}{$\frac{\text { Aderenza }}{\mathrm{G} 2}$} & \multirow[t]{2}{*}{ t-value } & \multirow[t]{2}{*}{ p-value } \\
\hline & & & & \\
\hline KDQOL & $89.17 \pm 16.32$ & $67.96 \pm 32.09$ & -2.144 & $0.013^{*}$ \\
\hline \multicolumn{5}{|l|}{ Dolore } \\
\hline BUT & $1.59 \pm 0.42$ & $2.35 \pm 0.70$ & $3.433 \mathrm{a}$ & $0.026 *$ \\
\hline \multicolumn{5}{|c|}{$\begin{array}{l}\text { Sintomo psicologico } \\
\text { Indice di angoscia }\end{array}$} \\
\hline BUT & $0.18 \pm 0.27$ & $0.60 \pm 0.78$ & $2348 \mathrm{a}$ & $0.002 *$ \\
\hline
\end{tabular}

I valori sono presentati come media $\pm \mathrm{SD} .{ }^{*} \mathrm{p}<0.05$ (t-test Student).

Sono state studiate le caratteristiche psicologiche secondo la conformità. Le pazienti che avevano aderito alle sei variabili descritte (attività fisica, esami medici, modelli dietetici di aderenza, assunzione di acqua, terapia farmacologica e pressione arteriosa controllata) sono state considerate conformi e assegnate al gruppo 1 (G1) mentre le pazienti che non avevano aderito anche a una singola variabile sono state considerate non conformi e assegnate al gruppo 2 (G2). Il gruppo G1 ha valori più alti per quanto riguarda il dolore e questo mostra un gruppo di pazienti che lamentano una sintomatologia dolorosa legata alla patologia da cui sono affette. Allo stesso tempo, il gruppo G1 riporta valori più bassi di angoscia relativi all'immagine corporea. Il gruppo G2 riporta alti valori di disagio relativi all'immagine corporea che portano il soggetto a mettere in atto una modalità difensiva o di evitamento rispetto a chi non può accettare sé stesso. I punteggi medi sono mostrati nella Tabella 4.

\section{Limitazioni dello studio}

Questo studio ha alcune limitazioni. La dimensione del campione era piccola e le partecipanti erano tutte reclutate in un singolo ospedale su una sola unità operativa, quindi è difficile generalizzare i risultati. Un altro limite è stato il periodo di trattamento limitato a 9 mesi. Studi futuri dovrebbero includere più ospedali, avere dimensioni del campione maggiori e un periodo di analisi più lungo.

\section{Implicazioni per la pratica infermieristica}

La recente letteratura sottolinea come il coinvolgimento dei pazienti rimane fondamentale per la riprogrammazione delle strategie nazionali e per affrontare efficacemente le malattie croniche a livello europeo. ${ }^{20}$ La cura e il trattamento della persona con ADPKD richiede un nuovo paradigma concettuale bio-psico-sociale, che ci permetta di osservare il paziente in una prospettiva avente tutti gli elementi di relazione ed esperienza che caratterizzano la sua malattia. Questo orientamento richiede una riflessione profonda sugli interventi educativi assistenziali utili a promuovere l'emancipazione e l'empowerment delle persone coinvolte. Allo stesso modo, la valutazione psicologica è importante perché consente una maggiore comprensione del benessere psicologico connesso all'aderenza alle terapie e permette di proporre gli interventi più utili. Recentemente, il concetto di "Patient Engagement" ha guadagnato l'attenzione delle istituzioni sanitarie; in altre parole, si può definire come "coinvolgimento attivo del paziente" che, negli ultimi decenni, ha portato ad una profonda trasformazione dei modelli sanitari verso una maggiore promozione del ruolo del paziente, visto come soggetto attivo ed "esperto" all'interno del processo clinico. Il coinvolgimento del paziente affetto da una malattia cronica è considerato un fattore importante per una buona aderenza ai trattamenti terapeutici. Aderenza terapeutica significa la misura in cui i pazienti seguono le istruzioni dei trattamenti terapeutici, ed è questo uno degli aspetti più difficili da gestire nei pazienti affetti da CKD. La non aderenza farmacologica può essere pericolosa per la salute dei pazienti e potrebbe comportare un peggioramento della progressione della CKD fino allo stadio finale della malattia. I vantaggi portati da questo studio potrebbero essere: a) maggiore conoscenza da parte del paziente della malattia, delle terapie e dei comportamenti positivi al fine di promuovere una gestione autonoma con benefici fisici e psicologici; b) modificare i comportamenti errati al fine di ottenere un equilibrio tra i bisogni del paziente e la malattia; c) migliorare l'osservanza delle terapie come risultato di un trattamento efficace e di una migliore qualità della vita; $\mathrm{d}$ ) ottenere e mantenere le capacità e le competenze per vivere nel miglior modo possibile il rapporto quotidiano con la patologia.

\section{Conclusioni}

Questo studio offre la possibilità di fare una prima valutazione psicologica delle donne con ADPKD, permettendo così di far emergere considerazioni preliminari relative al malessere psicologico che le pazienti 
possono provare. Lo studio mostra che le donne con ADPKD possono avere livelli lievi o moderati di ansia e depressione ed un declino della qualità della vita correlata alla salute. Esiste anche un'associazione tra preoccupazioni legate alla propria immagine corporea e sintomi ansioso-depressivi.

Ad ogni modo, lo studio mostra che i soggetti con aderenza hanno livelli più elevati di dolore fisico rispetto a quelli non aderenti. Tuttavia, il gruppo conforme ha meno disagio legato all'immagine corporea e ha meno uso di strategie di evitamento in relazione al disagio della propria immagine corporea.

Inoltre, per verifica del peggioramento della malattia, un'alta percentuale del campione studiato dimostra una buona pressione arteriosa e una corretta aderenza alle modificazioni dietetiche raccomandate (dieta a basso contenuto di sale e a basso contenuto proteico) anche se solo il $45 \%$ rispetta la raccomandazione di eseguire attività fisica. La soppressione dell'ormone antidiuretico con l'assunzione di alti quantitativi di acqua è un metodo noto per attenuare la progressione della crescita della cisti renale ed è questo un valido motivo per stimolare il paziente a garantire un'adeguata assunzione giornaliera di acqua. Per gli studi futuri prevediamo incontri con gruppi di pazienti condivisi dallo psicologo e dall'infermiere in modo da valorizzare le capacità comunicativo-relazionali di ogni figura professionale al fine di facilitare il processo terapeutico e rispondendo adeguatamente ai bisogni, ai dubbi, alle paure e le ansie, anche dei membri della famiglia, ottenendo un miglioramento della qualità della vita.

\section{Dichiarazione di assenza di conflitto di interessi}

Gli Autori dichiarano di non avere conflitti di interessi.

\section{Finanziamenti}

Gli Autori dichiarano di non aver ricevuto finanziamenti specifici da qualsiasi ente nei settori pubblico, privato o senza fini di lucro.

\section{Bibliografia}

1. Pei Y and Watnick T. Diagnosis and screening of autosomal dominant polycystic kidney disease. Advance in Chronic Kidney Disease. 2010; 17(2):140-152.

2. Chapman AB. Cystic disease in women: clinical characteristic and Medical Management. Advances in Renal Replacement Therapy. 2003; 10(1):24-33.

3. KDIGO. Clinical Practice Guideline for the Evaluation and Management of Chronic Kidney Disease, Kidney Int. 2012; (3): $1-150$.

4. Simms RJ, Thong KM, Dworschak GC, et al. Increased Psychosocial Risk, depression and reduced quality of life living with autosomal dominant polycystic kidney disease. Nephrol Dial Transplant. 2016; 31(7):1130-1140.
5. Lecourt N. Serious somatic disease as a limit-experience: clinical aspect of end-stage renal disease. Soins. 2018; 63(826):43-45.

6. Pierobon A, Giardini A, Callegari S, et al. Psychological adjustment to a chronic illness: the contribution from cognitive behavioral treatment in a rehabilitation setting. Giornale Italiano Medicina del Lavoro ed Ergonomia. 2011; 33(1):8-11.

7. Miskulin DC, Abebe KZ, Chapman AB, et al. Healthrelated quality of life in patients with autosomal dominant polycystic kidney disease and CKD stages 1- 4: a crosssectional study. Am J Kidney Dis. 2014; (63):214-226.

8. Pérez Domìnguez TS, Rodriguez Perez A, Buset Rios N, et al. Psychonephrology: psychological aspects in autosomal dominant polycystic kidney disease. Nephrology. 2011; (31):716-722.

9. Kimmel PL and Patel SS. Quality of life in patients with chronic kidney disease: focus on end-stage renal disease treated with hemodialysis. Semi Nephrol. 2006; (26): 68-79.

10. Tran W, Huynh D, Chan T, Chesla CA, et al. Understanding barriers to medication, dietary, and lifestyle treatments prescribed in polycystic kidney disease. BMC Nephrol. 2017; 18(5):214.

11. Cowley BD Jr. and Bissler JJ. Polycystic Kidney Disease. New York: Springer 2018.

12. WHO. Therapeutic Patient Education. Continuing Education Programs for Health Care Providers in the Field of Prevention of Chronic Diseases Report of a WHO Working Group. 1996.

13. Xavier F, Ferrazb M, Marcc N, et al. Elderly people's definition of quality of life. Rev Bras Psiquiatr. 2003; vol.25(1):31-39.

14. Burrai F, Hasan W, Luppi M, et al. A Conceptual Framework Encompassing the Psychoneuroimmunoendocrinological Influences of Listening to Music in Patients With Heart Failure. Holistic Nursing Practice. 2018; 32(2):81-89.

15. Miselli V. Il problema dell'adesione alla terapia in una malattia cronica come il diabete. G It Diabetol Metab. 2011; (31):121-124.

16. Chapman AB. Approaches to testing new treatments in autosomal dominant polycystic kidney disease: insights from the CRISP and HALT-PKD studies. Clin J Am Soc Nephrol. 2008; (3):1197-1204.

17. Klersy C, Callegari A, Giorgi I, et al. Italian translation, cultural adaptation and validation of KDQOL-SF, version 1.3, in patients with severe renal failure. Journal of Nephrology. 2007; 20(1):43-51.

18. Zigmond AS and Snaith RP. The hospital anxiety and depression scale. Acta Psychiatrica Scandinavica. 1983; 67(6):361-370.

19. Cuzzolaro M, Vetrone G, Marano G, et al. The Body Uneasiness Test (BUT): development and validation of a new body image assessment scale. Eating and Weight Disorders. 2006; 11(1):1-13.

20. Cordier JF. The expert patient: towards a novel definition Respiratory Journal. 2014; (44):853-857. 